\title{
Dynamic Programming Algorithms with Data Rounding for Combinatorial Food Packing Problems*
}

\author{
Yoshiyuki KARUNO**, Kazushi TAKAHASHI** and Atsushi YAMADA** \\ ** Department of Mechanical and System Engineering, Kyoto Institute of Technology \\ Matsugasaki, Sakyo-ku, Kyoto-shi, Kyoto 606-8585, Japan \\ E-mail: karuno@kit.ac.jp
}

\begin{abstract}
The lexicographic bi-criteria combinatorial food packing problem to be discussed in this paper is described as follows. Given a set $I=\{i \mid i=1,2, \ldots, n\}$ of current $n$ items (for example, $n$ green peppers) with their weights $w_{i}$ and priorities $\gamma_{i}$, the problem asks to find a subset $I^{\prime}(\subseteq I)$ so that the total weight $\Sigma_{i \in I^{\prime}} w_{i}$ is no less than a specified target weight $T$ for each package, and it is minimized as the primary objective, and further the total priority $\Sigma_{i \in I^{\prime}} \gamma_{i}$ is maximized as the second objective. The problem has been known to be NP-hard, while it can be solved exactly in $O(n T)$ time if all the input data are assumed to be integral. In this paper, we design a heuristic algorithm for the problem by applying a data rounding technique to an $O(n T)$ time dynamic programming procedure. We also conduct numerical experiments to examine the empirical performance such as execution time and solution quality.
\end{abstract}

Key words : Engineering Optimization, Food Manufacturing, Automatic Combination Weighers, Dynamic Programming, Data Rounding, Heuristic Algorithms.

\section{Introduction}

In this paper, we deal with a combinatorial optimization problem arising in actual food packing equipments, so-called automatic combination weighers, e.g., see Morinaka ${ }^{(10)}$. As depicted in Fig. 1, the automated food packing system typically consists of $n$ weighing hoppers, e.g., see Karuno, Nagamochi and Wang ${ }^{(8)}$. Some amount of foods (such as a green pepper, a ham, a handful of potato chips, and so on) is thrown into each hopper, and it is called an item. Given a set $I$ of current $n$ items in hoppers, the automated food packing system chooses a subset $I^{\prime}(\subseteq I)$ of items, and the chosen items are put into a single package. The resulting empty hoppers are supplied with next new items, and the set $I$ is updated by taking the union of the remaining items in $I-I^{\prime}$ and the new items. The packing operation is repeated to produce a large number of packages one by one.

Note that the automated food packing system always chooses some items in the current hoppers without knowing the weights of next new items. Some automatic combination weighers are able to produce nearly two hundred packages per minute at the maximum, i.e., approximately three hundred milliseconds per packing operation, e.g., see a manufacturer's site $^{(16)}$. The automatic combination weighers spend most time in measuring the weights of next new items accurately, and only a few milliseconds may be left for choosing a subset $I^{\prime}$ of items at each packing operation.

The total weight of chosen items for each package must be no less than a specified target weight from a viewpoint of the service conscience. The target weight constraint is a hard constraint of the problem of choosing a subset $I^{\prime}$, e.g., see again Morinaka ${ }^{(10)}$. Let $w_{i}$ denote the weight of item $i$, i.e., the current item in the $i$-th hopper, and let $T$ denote the target weight. Then, the target weight constraint is represented by $\Sigma_{i \in I^{\prime}} w_{i} \geq T$. The primary objective of 
each packing operation is to minimize $\Sigma_{i \in I^{\prime}} w_{i}$ under the target weight constraint. In other words, this aims at minimizing the amount of surplus in each package.

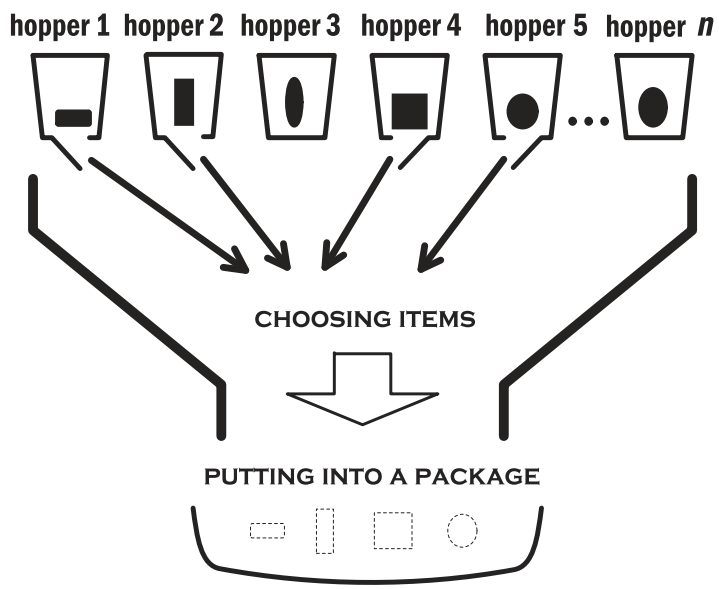

Fig. 1 Automated Food Packing System with $n$ Hoppers

During an operating run, i.e., a series of packing operations, an item may be left in hopper for a long time before it is chosen to be packed, e.g., see Kameoka and Nakatani ${ }^{(6)}$. In order to avoid such a situation, a priority $\gamma_{i}$ is introduced into each item $i$, which is defined by a non-decreasing function of the duration in hopper of the item, e.g., see again Karuno, Nagamochi and Wang ${ }^{(8)}$. Then, the total priority $\Sigma_{i \in I^{\prime}} \gamma_{i}$ is to be maximized as the second objective of each packing operation. We expect the items with longer durations in hoppers to be preferably chosen by the second objective.

The problem of choosing a subset $I^{\prime}$ from the set $I$ of current $n$ items with respect to the total weight and total priority objectives is regarded as a lexicographic bi-criteria 0-1 integer programming problem. Even with the total weight objective only, the combinatorial food packing problem is NP-hard, e.g., see Garey and Johnson ${ }^{(1)}$. Karuno, Nagamochi and Ohshima $^{(7)}$ showed that the combinatorial food packing problem can be solved in $O\left(n^{2} w_{\max }\right)$ time if all input data are integral, where $w_{\max }$ is the maximum of current $n$ weights. Afterward, Imahori, Karuno, Nagamochi and $\mathrm{Wang}^{(3)}$ improved the time complexity into $O(n T)$ with respect to the $n$, i.e., it is linear in $n$. These pseudo-polynomial time algorithms imply that the combinatorial food packing problem is not strongly NP-hard, but is weakly NP-hard, e.g., see again Garey and Johnson ${ }^{(1)}$. From the viewpoint of optimizing the total weight objective, some numerical results for automatic combination weighers were also reported, e.g., see Kameoka, Nakatani and Inui ${ }^{(5)}$, Murakami et al. ${ }^{(11)-(14)}$ (But the packing algorithms employed in their papers basically enumerate $O\left(2^{n}\right)$ feasible subsets $I^{\prime}$.)

As mentioned above, the previous papers have mainly investigated exact algorithms for the combinatorial food packing problem. In this paper, we design a heuristic algorithm for the combinatorial food packing problem by applying a data rounding technique to an $O(n T)$ time dynamic programming procedure. Roughly speaking, for a positive real $\delta$, by rounding the weights as $\left\lfloor w_{i} / \delta\right\rfloor(i=1,2, \ldots, n)$ and the target weight as $\lceil T / \delta\rceil$, the execution time is expected to be improved at the rate of nearly $1 / \delta$. On the other hand, the total weight objective can not always take the minimum, i.e., even the primary objective value of the total weight may involve a certain error from the minimum. In this paper, we also conduct numerical experiments to examine the empirical performance such as execution time and solution quality, and report the results.

\section{Problem Description}

Let $n$ denote the number of weighing hoppers in the automated food packing system to be treated in this paper (see again Fig. 1), and let $N$ denote the total number of packages to be 
produced during an operating run, which is equivalent to the number of iterations of packing operation during the operating run.

We call the combinatorial food packing problem with respect to the lexicographic bicriteria, i.e., the total weight of chosen items as the primary objective and total priority as the second objective, LEXICO for short. When we disregard the total priority, i.e., only with the primary objective of the total weight, we call the combinatorial food packing problem PRIMAL.

The current item in the $i$-th hopper is referred to as item $i$. Let $\ell$ denote the current iteration number of packing operation $(1 \leq \ell \leq N)$, and let $\ell_{i}$ denote the iteration number at which item $i$ has been thrown into the $i$-th hopper when the hopper was empty. Then, we refer to $d_{i}=\ell-\ell_{i}+1$ as the duration in hopper of item $i$. In this paper, we are going to set the priority of item $i$ to be the duration.

An instance of problem LEXICO at each packing operation consists of the following input data:

- $\quad I=\{i \mid i=1,2, \ldots, n\}$ : Set of current $n$ items.

- $w_{i}$ : Positive integer weight of item $i \in I$.

- $\gamma_{i}\left(:=d_{i}\right)$ : Positive integer priority of item $i \in I$.

- $\quad T$ : Target weight for each package, which is also assumed to be a positive integer.

For notational convenience, the maximum weight and the total weight over the $n$ items in $I$ are denoted by

$$
w_{\max }=\max _{1 \leq i \leq n}\left\{w_{i}\right\} \quad \text { and } \quad w_{\text {sum }}=\sum_{i=1}^{n} w_{i}
$$

respectively.

Problem LEXICO is formulated by using a 0 - 1 vector $x=\left(x_{1}, x_{2}, \ldots, x_{n}\right)$, instead of a subset $I^{\prime}$, where

$$
x_{i}= \begin{cases}1 & \text { if item } i \text { is chosen } \\ 0 & \text { otherwise }\end{cases}
$$

\section{LEXICO}

$$
\begin{array}{ll}
\text { minimize } & f(x)=\sum_{i=1}^{n} w_{i} x_{i} \quad \text { as the primary objective, } \\
\text { maximize } & g(x)=\sum_{i=1}^{n} \gamma_{i} x_{i} \quad \text { as the second objective, } \\
\text { subject to } & \sum_{i=1}^{n} w_{i} x_{i} \geq T \\
& x_{i} \in\{0,1\}, i=1,2, \ldots, n .
\end{array}
$$

The target weight constraint and binary constraints of variables $x_{i}$ are represented by Eqs. (5) and (6), respectively. A solution $x=\left(x_{1}, x_{2}, \ldots, x_{n}\right)$ satisfying Eqs. (5) and (6) is referred to as a feasible solution of problem LEXICO. The primary objective of Eq. (3) aims at attaining the total weight of chosen items in a feasible solution as close to the target weight $T$ as possible, together with Eq. (5) (i.e., the target weight constraint). The second objective of Eq. (4) is introduced so that the items with longer durations in hoppers are preferably chosen. (Recall that we do not solve the combinatorial food packing problem of on-line setting, but of off-line setting at each packing operation.) In order to omit some trivial cases ${ }^{(3)}$, we assume that

$$
w_{\max }<T \leq w_{\text {sum }},
$$

which implies $T \leq n \cdot w_{\max }$ (see Eq. (1)). 
For a given instance of problem LEXICO, let $f^{*}$ denote the minimum of the total weight of chosen items in a feasible solution, and let $x=\hat{x}$ denote a feasible solution that attains the minimum of the total weight, i.e., $f^{*}=f(\hat{x})$. An optimal solution $x=x^{*}$ is defined as a feasible solution such that it satisfies $f\left(x^{*}\right)=f^{*}$ and maximizes the total priority among feasible solutions with the minimum total weight $f^{*}$, i.e., it satisfies $g\left(x^{*}\right) \geq g(\hat{x})$ for any $\hat{x}$ with $f(\hat{x})=f^{*}$. We call $g^{*}=g\left(x^{*}\right)$ the conditionally maximum total priority. Problem LEXICO asks to find an optimal solution $x^{*}$. As mentioned before, if we are asked to find a feasible solution $x=\hat{x}$ with the minimum total weight $f^{*}$, we call the problem PRIMAL.

The following property of an optimal solution $x=x^{*}$ of problem LEXICO has been known, e.g., see Imahori, Karuno, Nagamochi and $\operatorname{Wang}^{(3)}$ :

Lemma 1. For an instance of problem LEXICO, the minimum total weight $f^{*}$ satisfies

$$
T \leq f^{*} \leq T+w_{\max }-1,
$$

where $T$ is the target weight and $w_{\max }$ is the maximum of the weights of current $n$ items.

In this paper, we are also going to incorporate the above property in the proposed heuristic algorithm. We now define the upper bound in Lemma 1 by

$$
b=T+w_{\max }-1 .
$$

Note that it holds $O(b)=O(T)$ by Eq. (7).

\section{Dynamic Programming}

In the $O(n T)$ time dynamic programming procedure which we are going to apply to the proposed heuristic algorithm, the following 0-1 state variables are defined for $k=1,2, \ldots, n$ and $p=0,1, \ldots, b$ :

$u_{k}(p)=1 \Longleftrightarrow\left\{\begin{array}{l}\text { There exists a (partial) } 0-1 \text { vector }\left(x_{1}, x_{2}, \ldots, x_{k}\right) \text { such that } \\ \sum_{i=1}^{k} w_{i} x_{i}=p .\end{array}\right.$

$u_{k}(p)=0 \Longleftrightarrow$ Such a $0-1$ vector does not exist.

The priority recoding variables $v_{k}(p)$ for $k=1,2, \ldots, n$ and $p=0,1, \ldots, b$ are also defined to maintain the total priority $\sum_{i=1}^{k} \gamma_{i} x_{i}$ of the $0-1$ vector $\left(x_{1}, x_{2}, \ldots, x_{k}\right)$ such that $\sum_{i=1}^{k} w_{i} x_{i}=p$. Further, two kinds of additional variables $\sigma_{k}(p)$ and $\tau_{k}(p)$ for $k=1,2, \ldots, n$ and $p=0,1, \ldots, b$ are introduced to make each priority recording variable $v_{k}(p)$ handle the conditionally maximum for the $0-1$ vector $\left(x_{1}, x_{2}, \ldots, x_{k}\right)$ such that $\sum_{i=1}^{k} w_{i} x_{i}=p$.

The $0-1$ state variables and priority recording variables are computed by the following dynamic programming recursives: For $p=0,1, \ldots, b$,

$$
\begin{aligned}
& u_{1}(p)= \begin{cases}1 & \text { if } p \in\left\{0, w_{1}\right\}, \\
0 & \text { otherwise, }\end{cases} \\
& v_{1}(p)=\left\{\begin{array}{cl}
0 & \text { if } p=0, \\
\gamma_{1} & \text { if } p=w_{1}, \\
-1 & \text { otherwise, }
\end{array}\right.
\end{aligned}
$$

and for $k=2,3, \ldots, n$ and $p=0,1, \ldots, b$,

$$
\begin{aligned}
& u_{k}(p)= \begin{cases}1 & \text { if }\left(u_{k-1}(p)=1\right), \\
\text { or if }\left(p-w_{k} \geq 0 \text { and } u_{k-1}\left(p-w_{k}\right)=1\right), & \text { otherwise, }\end{cases} \\
& \sigma_{k}(p)= \begin{cases}v_{k-1}(p) & \text { if } u_{k-1}(p)=1, \\
-1 & \text { otherwise, }\end{cases} \\
& \tau_{k}(p)= \begin{cases}v_{k-1}\left(p-w_{k}\right)+\gamma_{k} & \text { if }\left(p-w_{k} \geq 0 \text { and } u_{k-1}\left(p-w_{k}\right)=1\right), \\
-1 & \text { otherwise, }\end{cases} \\
& v_{k}(p)=\max \left\{\sigma_{k}(p), \tau_{k}(p)\right\} .
\end{aligned}
$$


In this paper, we call the above dynamic programming algorithm Base_DP. The correctness and time complexity ${ }^{(3)}$ are sketched as follows: From Eqs. (10)-(15), the computation of all variables $u_{k}(p), v_{k}(p), \sigma_{k}(p)$ and $\tau_{k}(p)$ requires $O(n b)=O(n T)$ time since it holds $O(b)=O(T)$ by assumption and definition (see Eq. (9)). After the computation, we find a minimum $p=p_{\min }$ such that $u_{n}(p)=1$ and $p \geq T$. We can see $p_{\min }=f^{*}$ (i.e., the minimum total weight), and the $p_{\min }$ can be found in additional $O(b)=O(T)$ time. We can also see $v_{n}\left(p_{\min }\right)=v_{n}\left(f^{*}\right)=g^{*}$ (i.e., the conditionally maximum total priority) from Eqs. (13)(15), where each $v_{k}(p)$ maintains the maximum of the total priority $\sum_{i=1}^{k} \gamma_{i} x_{i}$ of a $0-1$ vector $\left(x_{1}, x_{2}, \ldots, x_{k}\right)$ such that $\Sigma_{i=1}^{k} w_{i} x_{i}=p$ (if $\left.u_{k}(p)=1\right)$. Thus, algorithm Base_DP can compute the $f^{*}$ and $g^{*}$ in $O(n T)$ time. By backtracking the computation process, we can construct an optimal solution $x=x^{*}$.

In Table 1, we show the behavior of algorithm Base_DP for an example with five items, i.e., $n=5$. The entries of variables $u_{k}(p)$ and $v_{k}(p)$ are computed by Eqs. (10)-(15). For $k=1$, the setting of additional 0-1 variables $x_{k}(p)$ for the backtracking process is obvious. For $k \geq 2$, each entry $x_{k}(p)$ is set to be zero if it holds $u_{k}(p)=1$ due to $u_{k-1}(p)=1$, while it is set to be one if it holds $u_{k}(p)=1$ due to $p-w_{k} \geq 0$ and $u_{k-1}\left(p-w_{k}\right)=1$ (see Eq. (12)). If both conditions in Eq. (12) are satisfied, the $x_{k}(p)$ depends upon the choice of the corresponding $v_{k}(p)$ (see Eq. (15)), i.e., it is set to be zero if $v_{k}(p):=\sigma_{k}(p)$, while it is set to be one if $v_{k}(p):=\tau_{k}(p)$. Equation (15) breaks ties arbitrarily. We see that the minimum $p=p_{\min }$ is obtained as $p_{\min }=f^{*}=12(=T)$, and the conditionally maximum total priority is obtained as $g^{*}=v_{5}(12)=7$. The asterisks attached to the values of $u_{k}(p)$ indicate the backtracking process. Following them, an optimal solution $x^{*}=(1,0,0,1,0)$ is obtained. (A similar table is provided by Ibaraki ${ }^{(2)}$ for the so-called subset-sum problem.)

Table 1 Behavior of Base_DP for an Example

\begin{tabular}{|r|ccl|ccc|ccc|ccc|ccc|}
\hline$p$ & $u_{1}$ & $v_{1}$ & $x_{1}$ & $u_{2}$ & $v_{2}$ & $x_{2}$ & $u_{3}$ & $v_{3}$ & $x_{3}$ & $u_{4}$ & $v_{4}$ & $x_{4}$ & $u_{5}$ & $v_{5}$ & $x_{5}$ \\
\hline 0 & 1 & 0 & 0 & 1 & 0 & 0 & 1 & 0 & 0 & 1 & 0 & 0 & 1 & 0 & 0 \\
1 & 0 & -1 & - & 0 & -1 & - & 0 & -1 & - & 0 & -1 & - & 0 & -1 & - \\
2 & 0 & -1 & - & 0 & -1 & - & 1 & 1 & 1 & 1 & 1 & 0 & 1 & 1 & 0 \\
3 & 0 & -1 & - & 0 & -1 & - & 0 & -1 & - & 0 & -1 & - & 0 & -1 & - \\
4 & 0 & -1 & - & 0 & -1 & - & 0 & -1 & - & 1 & 2 & 1 & 1 & 2 & 0 \\
5 & 0 & -1 & - & 0 & -1 & - & 0 & -1 & - & 0 & -1 & - & 0 & -1 & - \\
6 & 0 & -1 & - & 0 & -1 & - & 0 & -1 & - & 1 & 3 & 1 & 1 & 3 & 0 \\
7 & 0 & -1 & - & 0 & -1 & - & 0 & -1 & - & 0 & -1 & - & 0 & -1 & - \\
8 & $1^{*}$ & 5 & 1 & $1^{*}$ & 5 & 0 & $1^{*}$ & 5 & 0 & 1 & 5 & 0 & 1 & 5 & 0 \\
9 & 0 & -1 & - & 0 & -1 & - & 0 & -1 & - & 0 & -1 & - & 0 & -1 & - \\
10 & 0 & -1 & - & 1 & 4 & 1 & 1 & 6 & 1 & 1 & 6 & 0 & 1 & 6 & 0 \\
11 & 0 & -1 & - & 0 & -1 & - & 0 & -1 & - & 0 & -1 & - & 0 & -1 & - \\
\hline 12 & 0 & -1 & - & 0 & -1 & - & 1 & 5 & 1 & $1^{*}$ & 7 & 1 & $1^{*}$ & 7 & 0 \\
\hline 13 & 0 & -1 & - & 0 & -1 & - & 0 & -1 & - & 0 & -1 & - & 0 & -1 & - \\
14 & 0 & -1 & - & 0 & -1 & - & 0 & -1 & - & 1 & 8 & 1 & 1 & 8 & 0 \\
15 & 0 & -1 & - & 0 & -1 & - & 0 & -1 & - & 0 & -1 & - & 0 & -1 & - \\
16 & 0 & -1 & - & 0 & -1 & - & 0 & -1 & - & 1 & 7 & 1 & 1 & 9 & 1 \\
17 & 0 & -1 & - & 0 & -1 & - & 0 & -1 & - & 0 & -1 & - & 0 & -1 & - \\
18 & 0 & -1 & - & 1 & 9 & 1 & 1 & 9 & 0 & 1 & 9 & 0 & 1 & 10 & 1 \\
19 & 0 & -1 & - & 0 & -1 & - & 0 & -1 & - & 0 & -1 & - & 0 & -1 & - \\
20 & 0 & -1 & - & 0 & -1 & - & 1 & 10 & 1 & 1 & 10 & 0 & 1 & 11 & 1 \\
21 & 0 & -1 & - & 0 & -1 & - & 0 & -1 & - & 0 & -1 & - & 0 & -1 & - \\
\hline
\end{tabular}

\section{Data Rounding}

The data rounding technique which we adopt divides a certain positive real $\delta$ into the current $n$ weights $w_{i}(i=1,2, \ldots, n)$ and target weight $T$. By this, the execution time of the proposed heuristic algorithm may be improved from that of algorithm Base_DP. (In Table 1, for example, by noting that all the weights of five items and target weight are even numbers, we can make algorithm Base_DP run faster to obtain the optimal solution $x^{*}=(1,0,0,1,0)$, 
i.e., by setting $\delta=2$, we can skip the computation of $u_{k}$ and $v_{k}$ for all odd values of $p$ to obtain the optimal solution.) In general, however, we have to permit the resulting solution to be an approximate solution, i.e., it may involve a certain error from the minimum total weight.

Let $\varepsilon>0$ denote a positive real which is a given parameter for the proposed heuristic algorithm. We define

$$
\delta=\frac{\varepsilon T}{n}
$$

which we call the rounding divisor. Then, we set the rounded weight of each item to be

$$
w_{i}^{\mathrm{R}}:=\left\lfloor\frac{w_{i}}{\delta}\right\rfloor \quad \text { for } \quad i=1,2, \ldots, n,
$$

and the rounded target weight to be

$$
T^{\mathrm{R}}:=\left\lceil\frac{T}{\delta}\right\rceil
$$

Hence, for a given $\varepsilon>0$, the rounded instance of a given instance of problem LEXICO consists of the following input data:

- $\quad I=\{i \mid i=1,2, \ldots, n\}$ : Set of current $n$ items (i.e., unchanged).

- $\quad w_{i}^{\mathrm{R}}$ : Rounded positive integer weight of item $i \in I$.

- $\quad \gamma_{i}\left(:=d_{i}\right)$ : Positive integer priority of item $i \in I$ (i.e., unchanged).

- $\quad T^{\mathrm{R}}$ : Rounded target weight for each package, which is also a positive integer.

Formally, the problem composed of the rounded instances is formulated as follows:

\section{ROUND_LEXICO}

$$
\begin{array}{ll}
\text { minimize } & f_{\mathrm{R}}(x)=\sum_{i=1}^{n} w_{i}^{\mathrm{R}} x_{i} \quad \text { as the primary objective, } \\
\text { maximize } & g_{\mathrm{R}}(x)=\sum_{i=1}^{n} \gamma_{i} x_{i} \quad \text { as the second objective, } \\
\text { subject to } & \sum_{i=1}^{n} w_{i}^{\mathrm{R}} x_{i} \geq T^{\mathrm{R}} \\
& x_{i} \in\{0,1\}, i=1,2, \ldots, n .
\end{array}
$$

A solution $x=\left(x_{1}, x_{2}, \ldots, x_{n}\right)$ satisfying Eqs. (21) and (22) is feasible in the sense of problem ROUND_LEXICO. Also, we say that it is feasible in the rounded instance. For the rounded instance, let $f_{\mathrm{R}}^{*}$ denote the minimum of the total rounded weight of chosen items in a feasible solution in the sense of problem ROUND_LEXICO, and let $x=x^{\prime}$ denote such a feasible solution that attains the minimum total rounded weight $f_{\mathrm{R}}^{*}$, i.e., $f_{\mathrm{R}}^{*}=f_{\mathrm{R}}\left(x^{\prime}\right)$. As for problem LEXICO, if we disregard the total priority, we call the corresponding problem ROUND_PRIMAL. Let $w_{\max }^{\mathrm{R}}=\max _{1 \leq i \leq n} w_{i}^{\mathrm{R}}$. By Lemma 1, we straightforwardly obtain the following property:

Lemma 2. For an instance of problem ROUND_LEXICO, the minimum total rounded weight $f_{\mathrm{R}}^{*}$ satisfies

$$
T^{\mathrm{R}} \leq f_{\mathrm{R}}^{*} \leq T^{\mathrm{R}}+w_{\max }^{\mathrm{R}}-1
$$

where $T^{\mathrm{R}}$ is the rounded target weight and $w_{\max }^{\mathrm{R}}$ is the maximum of the rounded weights of current $n$ items.

We call the proposed heuristic algorithm Round_DP. For a given instance of problem LEXICO, algorithm Round_DP first computes the rounded weights $w_{i}^{\mathrm{R}}(i=1,2, \ldots, n)$ and rounded target weight $T^{\mathrm{R}}$, using a given parameter $\varepsilon>0$ (see Eqs. (16), (17) and (18)). Then, it solves the rounded instance by calling algorithm Base_DP. Finally, it outputs the solution $x=x^{\prime}$ that attains the minimum total rounded weight $f_{\mathrm{R}}^{*}$ as an approximate solution of 
the original instance of problem LEXICO. In the remainder of this section, we analyze the theoretical approximation guarantee of algorithm Round_DP.

For employing the solution $x^{\prime}$ as an approximate solution of the original instance of problem LEXICO, the following property is necessary:

Lemma 3. For a given instance of problem LEXICO, let $x^{\prime}=\left(x_{1}^{\prime}, x_{2}^{\prime}, \ldots, x_{n}^{\prime}\right)$ denote a feasible solution in the rounded instance. Then, the solution $x^{\prime}$ is also feasible in the original instance of problem LEXICO, i.e., it satisfies

$$
f\left(x^{\prime}\right)=\sum_{i=1}^{n} w_{i} x_{i}^{\prime} \geq T
$$

Proof. From Eqs. (17) and (18) which define the rounded wights and rounded target weight, we have

$$
0 \leq w_{i}-\delta w_{i}^{\mathrm{R}}<\delta \quad \text { and } \quad 0 \leq \delta T^{\mathrm{R}}-T<\delta .
$$

Hence, the total weight of chosen items in the solution $x^{\prime}$ satisfies

$$
\begin{aligned}
\sum_{i=1}^{n} w_{i} x_{i}^{\prime} & \geq \delta \sum_{i=1}^{n} w_{i}^{\mathrm{R}} x_{i}^{\prime} & & \text { (by Eq. (25)) } \\
& \geq \delta T^{\mathrm{R}} & & \text { (by definition and Eq. (21)) } \\
& \geq T, & & \text { (by Eq. (25)) }
\end{aligned}
$$

which implies the lemma.

Let $b^{\mathrm{R}}=T^{\mathrm{R}}+w_{\max }^{\mathrm{R}}-1$ denote the upper bound in Lemma 2. Then, we have $O\left(b^{\mathrm{R}}\right)=$ $O(T / \delta)=O(n / \varepsilon)$ since it holds $b^{\mathrm{R}}<\left(T+w_{\max }\right) / \delta<2 n / \varepsilon$ (see Eqs. (7), (16) and (25)), which implies that the rounded instance can be solved in $O\left(n b^{\mathrm{R}}\right)=O(n T / \delta)=O\left(n^{2} / \varepsilon\right)$ time by calling algorithm Base_DP. In other words, algorithm Round_DP runs in $O(n T / \delta)=O\left(n^{2} / \varepsilon\right)$ time, and outputs a feasible solution $x=x^{\prime}$ in the rounded instance (if it exists) which attains the minimum total rounded weight $f_{\mathrm{R}}^{*}$. We also see the next property:

Lemma 4. For a given instance of problem LEXICO and for a given real $\varepsilon>0$, let $f^{*}$ denote the minimum total weight attained by an optimal solution $x^{*}=\left(x_{1}^{*}, x_{2}^{*}, \ldots, x_{n}^{*}\right)$, i.e., $f^{*}=f\left(x^{*}\right)$, and let $x^{\prime}=\left(x_{1}^{\prime}, x_{2}^{\prime}, \ldots, x_{n}^{\prime}\right)$ denote a feasible solution obtained by algorithm Round DP in $O\left(n^{2} / \varepsilon\right)$ time. Then, if the optimal solution $x^{*}$ is a feasible solution in the rounded instance, the total weight of chosen items in the solution $x^{\prime}$ satisfies

$$
f\left(x^{\prime}\right)=\sum_{i=1}^{n} w_{i} x_{i}^{\prime}<(1+\varepsilon) f^{*}
$$

Proof. By assumption of $x^{*}$ in the rounded instance, it holds $f_{\mathrm{R}}\left(x^{*}\right) \geq f_{\mathrm{R}}\left(x^{\prime}\right)\left(\geq T^{\mathrm{R}}\right)$, i.e., there is the following relationship between the two feasible solutions $x^{*}$ and $x^{\prime}$ :

$$
\sum_{i=1}^{n} w_{i}^{\mathrm{R}} x_{i}^{*} \geq \sum_{i=1}^{n} w_{i}^{\mathrm{R}} x_{i}^{\prime} .
$$

Hence, together with Eqs. (8), (16) and (25), we have

$$
\begin{aligned}
\sum_{i=1}^{n} w_{i} x_{i}^{\prime} & <\sum_{i=1}^{n}\left(\delta w_{i}^{\mathrm{R}}+\delta\right) x_{i}^{\prime} & & \text { (by Eq. (25)) } \\
& \leq \delta \sum_{i=1}^{n} w_{i}^{\mathrm{R}} x_{i}^{*}+\delta \sum_{i=1}^{n} x_{i}^{\prime} & & \text { (by Eq. (27)) } \\
& \leq \sum_{i=1}^{n}\left(\delta w_{i}^{\mathrm{R}}\right) x_{i}^{*}+\delta n & & \\
& \leq \sum_{i=1}^{n} w_{i} x_{i}^{*}+\varepsilon T & & \text { (by Eqs. (16) and (25)) } \\
& \leq f^{*}+\varepsilon f^{*}, & & \text { (by Eq. (8)) }
\end{aligned}
$$


which completes the proof.

Eventually, we obtain the following bound on the total weight of chosen items in the approximate solution $x=x^{\prime}$ obtained by the proposed heuristic algorithm:

Theorem 1. For a given instance of problem LEXICO and for a given real $\varepsilon>0$, let $f^{*}$ denote the minimum total weight attained by an optimal solution $x^{*}=\left(x_{1}^{*}, x_{2}^{*}, \ldots, x_{n}^{*}\right)$, i.e., $f^{*}=f\left(x^{*}\right)$, and let $x^{\prime}=\left(x_{1}^{\prime}, x_{2}^{\prime}, \ldots, x_{n}^{\prime}\right)$ denote a feasible solution obtained by algorithm Round_DP in $O\left(n^{2} / \varepsilon\right)$ time. Then, the total weight of chosen items in the solution $x^{\prime}$ satisfies

$$
T \leq f\left(x^{\prime}\right)<(2+\varepsilon) f^{*} .
$$

Proof. It has been left to analyze the case of $f_{\mathrm{R}}\left(x^{*}\right)<T^{\mathrm{R}}$, i.e., the optimal solution $x^{*}$ is infeasible in the rounded instance. The relationship of Eq. (27) between the two solutions $x^{*}$ and $x^{\prime}$ does not hold in this case, and we employ Lemma 2 as another bound on the minimum total rounded weight $f_{\mathrm{R}}^{*}$. Then, we have

$$
\begin{aligned}
& \sum_{i=1}^{n} w_{i} x_{i}^{\prime}<\sum_{i=1}^{n}\left(\delta w_{i}^{\mathrm{R}}+\delta\right) x_{i}^{\prime} \quad \text { (by Eq. (25)) } \\
& \leq \delta f_{\mathrm{R}}^{*}+\delta n \\
& \leq \delta\left(T^{\mathrm{R}}+w_{\max }^{\mathrm{R}}-1\right)+\delta n \quad \text { (by Lemma 2) } \\
& <(T+\delta)+w_{\max }-\delta+\delta n \quad \text { (by Eq. (25)) } \\
& =T+w_{\max }+\varepsilon T \quad \text { (by Eq. (16)) } \\
& \leq(1+\varepsilon) f^{*}+w_{\max },
\end{aligned}
$$

which implies Eq. (28) in the theorem since it holds $f^{*} \geq T>w_{\max }$ by Eqs. (7) and (8), together with Lemma 3 and Lemma 4.

In this section, we implicitly assume that the rounded instance of a given instance of problem LEXICO has at least one feasible solution, i.e., $T^{\mathrm{R}} \leq \sum_{i=1}^{n} w_{i}^{\mathrm{R}}$. In order to maintain the theoretical approximation guarantee, the proposed heuristic algorithm would choose all $n$ items, using the rounding divisor $\delta=\varepsilon T /(n+1)$ instead of Eq. (16) if $T^{\mathrm{R}}>\sum_{i=1}^{n} w_{i}^{\mathrm{R}}$. (Recall that it is assumed that $\sum_{i=1}^{n} w_{i} \geq T$ by Eq. (1).)

The approximation guarantee $(2+\varepsilon)$ of the proposed heuristic algorithm is much interesting from the theoretical point of view, e.g., see Vazirani ${ }^{(15)}$, while it is hard for us to state that the approximation ratio is sufficiently small for the automatic combination weighers. However, it may hold $T \gg w_{\max }$ in practice. In the next section, we are going to examine the empirical performance of the proposed heuristic algorithm.

\section{Numerical Results}

The instances of problem LEXICO to be tested are randomly generated as follows:

- The number of hoppers: $n \in\{10,20,40\}$.

- Integer weights: $w_{i}$ 's are uniformly random integers in [150, 250].

- Target weight: $T=800$.

- The number of iterations of packing operations: $N=10000$.

In addition, the given real $\varepsilon>0$ to the proposed heuristic algorithm Round_DP is chosen from $\{0.05,0.1\}$. For example, given $n=20, T=800$ and $\varepsilon=0.05$, we have $\delta=2$ as the rounding divisor (see Eq. (16)). We remark that given $n=40, T=800$ and $\varepsilon=0.05$, we have $\delta=1.0$ as the rounding divisor, and hence algorithm Round DP is equivalent to algorithm Base_DP in this case.

The program is written in C. It is complied by Microsoft Visual C++ 2008 Express Edition, and run on a laptop computer with Windows 7 Home Premium (64bit), Intel Core i5 $2410 \mathrm{CPU}(2.3 \mathrm{GHz})$ and 4GB memory. Note that by giving a constant priority to each item 
in algorithm Base_DP (resp., algorithm Round_DP), we can equivalently solve the instances of problem PRIMAL (resp., of problem ROUND_PRIMAL).

In all the tables, each of the data indicates the mean value for ten operating runs of producing $N$ packages (and hence, $10,000 \times 10$ packages are produced to obtain each of the data). The notations used in the tables have the following meanings:

- CPU : The execution time of each algorithm required to obtain either an optimal or an approximate solution at each packing operation.

- $\quad$ NET : The mean value of the total weight over $N$ packages.

- $D_{\max }$ : The maximum duration over all items thrown into the automated food packing system during an operating run.

- $D_{\text {mean }}$ : The mean duration over all items thrown into the automated food packing system during an operating run.

Table 2 Execution Time of the Proposed Heuristic [msec]

\begin{tabular}{cccc}
\hline \hline & Base_DP & \multicolumn{2}{c}{ Round_DP } \\
& (an Exact Algorithm) & $\varepsilon=0.05$ & $\varepsilon=0.1$ \\
\hline \hline$n$ & & CPU for problem LEXICO & \\
\hline 10 & 0.16 & 0.08 & 0.06 \\
20 & 0.34 & 0.20 & 0.12 \\
40 & 0.67 & 0.67 & 0.38 \\
\hline \hline$n$ & & CPU for problem PRIMAL & 0.06 \\
\hline 10 & 0.16 & 0.08 & 0.12 \\
20 & 0.33 & 0.19 & 0.35 \\
40 & 0.64 & 0.64 & $w_{i} \in[150,250], T=800$
\end{tabular}

Table 2 shows the execution times of algorithms Base_DP and Round_DP. In the implementation, the algorithms include the dynamic programming procedure and some initializing steps as well to update the set of current $n$ items at each packing operation. Further, algorithm Round_DP includes the step of computing the rounded weights and rounded target weight. We observe the decrease in the cases of problem LEXICO with $n=10$ and $n=20$, but the rates are not so close to the $1 / \delta$, since the time required by the initializing steps and data rounding step at each packing operation is relatively large in the cases. In the case of problem LEXICO with $n=40$ and $\varepsilon=0.1$ (hence $\delta=2$ ), the execution time reduces from that of algorithm Base_DP at the rate of 0.38/0.67=0.57, which becomes near the $1 / \delta=0.5$. We also observe that algorithm Round_DP runs in the test cases of problem PRIMAL as in those of problem LEXICO with respect to the execution time.

Table 3 Total Weight of a Package Obtained by the Proposed Heuristic

\begin{tabular}{lccc}
\hline \hline & Base_DP & \multicolumn{2}{c}{ Round_DP } \\
& (an Exact Algorithm) & $\varepsilon=0.05$ & $\varepsilon=0.1$ \\
\hline \hline$n$ & NET by solving problem LEXICO \\
\hline 10 & 804.1 & 808.4 & 815.6 \\
20 & 801.4 & 802.9 & 806.4 \\
40 & 800.5 & 800.5 & 802.2 \\
\hline \hline$n$ & NET by solving problem PRIMAL \\
\hline 10 & 804.1 & 808.5 & 815.8 \\
20 & 802.0 & 803.7 & 807.1 \\
40 & 801.8 & 801.8 & 803.5 \\
\hline \hline
\end{tabular}

Table 3 shows the results on the total weight of chosen items in an optimal solution and an approximate solution obtained by algorithms Base_DP and Round_DP, respectively. We observe that the total weight obtained by algorithm Round_DP includes some error from the minimum total weight in each case (except for the case of problem LEXICO with $n=40$ and 
$\varepsilon=0.05$ ). However, the relative difference from the target weight (i.e., $T=800$ ) is about 2 [\%] at the maximum (see the case of problem LEXICO with $n=10$ and $\varepsilon=0.1$ ). In the case of problem LEXICO with $n=40$ and $\varepsilon=0.1$, the error is only about 0.3 [\%], which is so small from the viewpoint of the theoretical approximation guarantee. In this table, we also observe that algorithm Round_DP performs in the test cases of problem PRIMAL as in those of problem LEXICO.

Table 4 Maximum and Mean Durations

\begin{tabular}{cccc}
\hline \hline & Base_DP & \multicolumn{2}{c}{ Round_DP } \\
& (an Exact Algorithm) & $\varepsilon=0.05$ & $\varepsilon=0.1$ \\
\hline \hline$n$ & \multicolumn{2}{c}{$\left[D_{\max }\right]$ and $\left(D_{\text {mean }}\right)$ by problem LEXICO } \\
\hline 10 & {$[44.7](2.5)$} & {$[25.3](2.5)$} & {$[15.5](2.4)$} \\
20 & {$[66.2](5.0)$} & {$[50.0](5.0)$} & {$[32.8](5.0)$} \\
40 & {$[81.2](10.0)$} & {$[81.2](10.0)$} & {$[56.1](9.9)$} \\
\hline \hline$n$ & {$\left[D_{\max }\right]$ and $\left(D_{\text {mean }}\right)$ by problem PRIMAL } \\
\hline 10 & {$[64.3](2.5)$} & {$[100.6](2.5)$} & {$[121.7](2.4)$} \\
20 & {$[1929](5.0)$} & {$[5780](5.0)$} & {$[7199](4.9)$} \\
40 & {$[9063](9.9)$} & {$[9063](9.9)$} & {$[9882](9.9)$} \\
\hline \hline
\end{tabular}

Table 4 shows the maximum durations $D_{\max }$ and mean durations $D_{\text {mean }}$ obtained by algorithms Base_DP and Round_DP. The $D_{\max }$ obtained by algorithm Round_DP is less than 100 in each test case of problem LEXICO and it is also less than that obtained by algorithm Base_DP. On the other hand, the $D_{\max }$ obtained by algorithm Round_DP is more than 100 in each test case of problem PRIMAL. In particular, in the cases of problem PRIMAL with $n=20$ and $n=40$, each $D_{\max }$ exceeds 5000 . We have observed some similar results on the previous exact algorithms, one of which is algorithm Base_DP. That is, we see that the second objective of the total priority is effective for reducing the maximum duration $D_{\max }$ in algorithm Round_DP as well as algorithm Base_DP.

\section{Concluding Remarks}

Given a set $I=\{i \mid i=1,2, \ldots, n\}$ of current $n$ items (for example, $n$ green peppers) with their weights $w_{i}$ and priorities $\gamma_{i}$, the lexicographic bi-criteria combinatorial food packing problem asks to find a subset $I^{\prime}(\subseteq I)$ so that the total weight $\Sigma_{i \in I^{\prime}} w_{i}$ is no less than a specified target weight $T$ for each package, and it is minimized as the primary objective, and further the total priority $\sum_{i \in I^{\prime}} \gamma_{i}$ is maximized as the second objective. The problem has been known to be NP-hard, while it can be solved exactly in $O(n T)$ time if all the input data are assumed to be integral. In this paper, we designed a heuristic algorithm for the problem by applying a data rounding technique to an $O(n T)$ time dynamic programming procedure, and showed that for a given real $\varepsilon>0$, it delivers an approximate solution with factor $(2+\varepsilon)$ of the total weight objective in $O\left(n^{2} / \varepsilon\right)$ time. We also conducted numerical experiments to examine the empirical performance, and reported that the execution time was improved, and that the error of the total weight was not so large as the theoretical approximation guarantee. In fact, the relative difference of the total weight obtained by the proposed heuristic algorithm from the target weight was at most $2[\%]$ in all the test instances.

The theoretical approximation guarantee of more than two of the proposed heuristic algorithm seems to be large from the practical point of view, although the relative difference from the target weight was empirically small. In addition, the approximate solution obtained by the proposed heuristic algorithm has no theoretical guarantee with the second objective of the total priority. Therefore, it would be significant to design a heuristic algorithm with a better approximation guarantee for future research. Of course, it would be interesting to examine the proposed heuristic algorithm by more test instances with wider ranges. It would also be interesting to investigate whether the data rounding technique is convertible into the combinatorial food packing problems of the duplex and double-layered types of the automated food packing systems ${ }^{(4),(9)}$. 


\section{Acknowledgment}

The authors are grateful to an anonymous referee and the editor for their helpful comments. This research was partially supported by a Grant-in-Aid for Scientific Research (C) of the Japan Society for the Promotion of Science (JSPS).

\section{References}

( 1 ) Garey, M.R. and Johnson, D.S., Computers and Intractability: A Guide to the Theory of NP-Completeness, (1979), W.H. Freeman, San Francisco.

( 2 ) Ibaraki, T., Algorithms and Data Structures in C (in Japanese), (1999), Shokodo, Tokyo.

( 3 ) Imahori, S., Karuno, Y., Nagamochi, H. and Wang, X., Kansei engineering, humans and computers: efficient dynamic programming algorithms for combinatorial food packing problems, International Journal of Biometrics, Vol. 3, No. 3, (2011), pp. 228-245.

( 4 ) Imahori, S., Karuno, Y. and Yoshimoto, Y., Dynamic programming algorithms for duplex food packing problems, Proceedings of the 8th IEEE International Conference on Industrial Informatics, INDIN 2010, (2010), pp. 857-862.

( 5 ) Kameoka, K., Nakatani, M. and Inui, N., Phenomena in probability and statistics found in a combinatorial weigher (in Japanese), Transactions of the Society of Instrument and Control Engineers, Vol. 36, No. 5, (2000), pp. 388-394.

( 6 ) Kameoka, K. and Nakatani, M., Feed control criterion for a combination weigher and its effects (in Japanese), Transactions of the Society of Instrument and Control Engineers, Vol. 37, No. 10, (2001), pp. 911-915.

( 7 ) Karuno, Y., Nagamochi, H. and Ohshima, Y., A dynamic programming approach for a food packing problem (in Japanese), Transactions of the Japan Society of Mechanical Engineers, Series C, Vol. 72, No. 716, (2006), pp. 1390-1397.

( 8 ) Karuno, Y., Nagamochi, H. and Wang, X., Bi-criteria food packing by dynamic programming, Journal of the Operations Research Society of Japan, Vol. 50, No. 4, (2007), pp. 376-389.

( 9 ) Karuno, Y., Nagamochi, H. and Wang, X., Optimization problems and algorithms in double-layered food packing systems, Journal of Advanced Mechanical Design, Systems, and Manufacturing, Vol. 4, No. 3, (2010), pp. 605-615.

(10) Morinaka, H., Automatic combination weigher for product foods (in Japanese), Journal of the Japan Society of Mechanical Engineers, Vol. 103, No. 976, (2000), pp. 130-131.

(11) Murakami, Y., Kurata, J., Uchiyama, H. and Ueno, T., Characterization of infeasible solutions in a bag-packing problem for achieving desired weight (in Japanese), Transactions of the Society of Instrument and Control Engineers, Vol. 38, No. 9, (2002), pp. 784-791.

(12) Murakami, Y., Kurata, J., Uchiyama, H. and Kawai, M., Raising the rate of feasible solutions in a bag-packing problem (by dividing input materials into groups of lighter items and heavier ones) (in Japanese), Transactions of the Japan Society of Mechanical Engineers, Series C, Vol. 69, No. 686, (2003), pp. 2830-2837.

(13) Murakami, Y., Kurata, J., Uchiyama, H., Taniya, K. and Kawai, M., Efficient algorithm for solving a bag-packing problem by excluding search space (in Japanese), Transactions of the Japan Society of Mechanical Engineers, Series C, Vol. 69, No. 688, (2003), pp. 3431-3438.

(14) Murakami, Y., Uchiyama, H., Kurata, J. and Kotera, T., High efficiency of weighing machine for achieving desired weights by tuning input amount (in Japanese), Transactions of the Japan Society of Mechanical Engineers, Series C, Vol. 74, No. 743, (2008), pp. 1920-1925.

(15) Vazirani, V.V., Approximation Algorithms, (2001), Springer, Berlin.

(16) Ishida Co., Ltd., http: //www. ishida.co.jp, Weighing and Packaging (in Japanese), [accessed July 2012]. 